\title{
Alta formazione professionale tra formal e non formal learning: il caso dei Master Universitari italiani
}

\section{Maddalena Formicuzzi, Serena Cubico, Jocilene Gadioli De Oliveira, Giuseppe Favretto, Anastasia Ferrari e Piermatteo Ardolino}

Il presente lavoro prende spunto dalla realizzazione del progetto di ricercalintervento "Master Universitari e competenze acquisite. Validazione e certificazione» finanziato dalla Regione Veneto. Il progetto ha consentito di realizzare una serie di osservazioni e riflessioni sulle metodologie didattiche, sugli strumenti di valutazione e di certificazione legate sia agli apprendimenti realizzati in ambito formale che a quelli non formali.

\section{Quadro teorico - istituzionale: Tra Formal e non Formal Learning}

Questo lavoro ha come obiettivo la presentazione dei passi compiuti per la realizzazione del progetto "Master universitari e competenze acquisiste. Validazione e certificazione» al fine di illustrarne gli strumenti utilizzati, gli attori coinvolti ed i risultati raggiunti e di condividere, attraverso essi, la necessità emersa di un chiarimento sui termini Formal e Non Formal Learning che, all'interno di percorsi professionalizzanti rischiano di perdere i propri confini concettuali e derivanti dalle definizioni espresse dall'Unione Europea richiedendo a progettisti e formatori uno sforzo metodologico e realizzativo privo di espliciti punti di riferimento.

Quando, nel 2000, l'Unione Europea formalizza la necessità che la formazione, fino a quel momento improntata solo al riconoscimento dell'apprendimento formale, si aprisse all'apprendimento informale e non formale, (Commissione delle Comunità Europee, 2000) introduce il concetto di formazione permanente e prova a formalizzare la distinzione tra i tre tipi di apprendimento creando un collegamento diretto con il luogo di formazione

Si distinguono tre diverse categorie fondamentali di apprendimento finalizzato:

l'apprendimento formale che si svolge negli istituti d'istruzione e di formazione e porta all'ottenimento di diplomi e di qualifiche riconosciute; 
l'apprendimento non formale che si svolge al di fuori delle principali strutture d'istruzione e di formazione e, di solito, non porta a certificati ufficiali. L'apprendimento non formale è dispensato sul luogo di lavoro o nel quadro di attività di organizzazioni o gruppi della società civile (associazioni giovanili, sindacati o partiti politici). Può essere fornito anche da organizzazioni o servizi istituiti a complemento dei sistemi formali (quali corsi d'istruzione artistica, musicale e sportiva o corsi privati per la preparazione degli esami);

l'apprendimento informale è il corollario naturale della vita quotidiana. Contrariamente all'apprendimento formale e non formale, esso non è necessariamente intenzionale e può pertanto non essere riconosciuto, a volte dallo stesso interessato, come apporto alle sue conoscenze e competenze. (Commissione delle Comunità Europee, 2000, pag. 9)

Fino a quel momento, l'istruzione formale aveva dominato la riflessione politica, influenzando l'impostazione dei modelli d'istruzione e formazione, nonché la percezione generale di «apprendimento». L'apprendimento permanente, senza soluzioni di continuità, avrebbe dovuto consentire l'inserimento dell'apprendimento non formale ed informale in un unico flusso di costruzione del «cittadino europeo» nel rispetto degli obiettivi comunitari e nel contempo della libertà ed individualità del singolo. (Commissione delle Comunità Europee, 2000)

Con questa impostazione e dichiarazione l'Unione Europea sembra aver metabolizzato quel concetto di «capacitazione» di cui parla Sen nel 1993 inteso come la possibilità per l'individuo di essere messo nella condizione di acquisire le «abilità di fare cose» e di «funzionare» in cui si riflette la libertà del singolo di poter scegliere e determinare in che direzione ed in quale contesto realizzare se stesso (Sen, 1993, 1999).

La capacità è dunque un insieme di vettori di funzionamenti, e riflette la libertà dell'individuo di condurre un tipo di vita piuttosto che un altro. Così come il cosiddetto «insieme di bilancio» nello spazio delle merci rappresenta la libertà della persona di comprare panieri di merci, l'«insieme delle capacità» nello spazio dei funzionamenti riflette la libertà della persona di scegliere fra le vite possibili. (Sen, 1993, pag. 38).

Queste parole richiamano e spiegano il senso della formazione continua e della necessità, per un pieno sviluppo e libertà dell'individuo, di consentire una capitalizzazione delle esperienze che non sia unicamente la capacità di cogliere ciò che di pratico esiste nelle attività formative, ma di valorizzare anche gli aspetti più legati all'individualità ed al diretto incontro con il mondo esterno, inteso nella sua definizione più ampia. Riprendendo un linguaggio più affine a quello dell'Unione europea si potrebbe dire

Se l'apprendimento lungo l'arco della vita è senza soluzioni di continuità, ciò mette in luce la complementarietà dell'apprendimento formale, non formale ed informale, il fatto che si possano acquisire conoscenze utili in diverse situazioni, contesti e momenti della vita. (Gola, 2009, pag. 61) 
La pratica strutturata diventa un sapere complementare alla teoria e ne acquisisce pari dignità; si recuperano il concetto di cooperative learning di peer learning ed experiential learning sviluppati a partire dalla fine degli anni '70 e si strutturano i concetti di competenze, certificazione, valutazione/validazione (Trentin 2000; Midoro, 2002).

Le indicazioni dell'Unione Europea ed il quadro concettuale fin qui delineato, hanno portato i paesi membri ad applicare una serie di riforme nei percorsi educativi e formativi istituzionali (e quindi formali) introducendo e rafforzando l'importanza del concetto dell'alternanza "scuola/lavoro» (Senato della Repubblica Italiana, 2010) che hanno portato alla strutturazione dei percorsi di formazione professionale come processo di formazione formale attraverso delle esperienze tipiche della formazione non formale (esperienze in azienda).

Il forte impatto assunto dalla "pratica professionale» nell'ambito della formazione formale, relativa ai gradi più alti della stessa (Scuole Superiori e Università), ha spinto l'Unione Europea a rivedere i concetti e la collocazione delle tipologie di formazione previste. Tali revisioni, che noi focalizziamo sugli aspetti della formazione formale e non formale per coerenza con i temi fin qui descritti, sono stati pubblicati dall'Unione Europea nel «Convalida dell'apprendimento non formale e informale». Si definisce:

- apprendimento formale: apprendimento erogato in un contesto organizzato e strutturato, specificamente dedicato all'apprendimento, che di norma porta all'ottenimento di qualifiche, generalmente sotto forma di certificati o diplomi; comprende sistemi di istruzione generale, formazione professionale iniziale e istruzione superiore;

- apprendimento non formale: apprendimento erogato mediante attività pianificate (in termini di obiettivi e tempi di apprendimento) con una qualche forma di sostegno all'apprendimento (ad esempio la relazione studente-docente); può comprendere programmi per il conseguimento di abilità professionali, alfabetizzazione degli adulti e istruzione di base per chi ha abbandonato la scuola prematuramente;. (Consiglio dell'Unione Europea, 2012, pag. 5)

In questo documento, per la prima volta, l'Unione Europea disgiunge la tipologia di formazione dal luogo in cui questa viene erogata, assegnando di fatto alla formazione formale la possibilità di rilasciare attestati, recependo per forma e sostanza la necessità di rispettare i Descrittori di Dublino (Ministry of Science, Technology and Innovation, 2005).

Alla formazione non formale viene quindi lasciata la possibilità di formare le abilità professionali, nel rispetto della strutturazione dei livelli del Quadro Europeo delle Qualifiche (Parlamento Europeo, 2008) ma non esplicita le possibili, ed a volte necessarie sovrapposizioni tra queste due tipologie di formazione. In sintesi: cosa succede in quei percorsi che contengono, di fatto, entrambi i tipi di formazione? Come coniugare due tipologie di formazione che portano a riconoscimenti diversi sul mercato e nel mondo dell'istruzione? Può un percorso di formazione formale, che sia costituito da un aspetto di formazione 
non formale, consentire una valutazione efficace delle competenze acquisite dagli allievi e produrre una certificazione efficace che consenta di fatto la capitalizzazione e trasferibilità delle esperienze?

\section{Master Universitari Italiani struttura e complessità}

I master universitari in Italia, hanno la caratteristica di essere «corsi di perfezionamento scientifico e di alta formazione ricorrente e permanente successivi al conseguimento della laurea o della laurea specialisticalmagistrale al termine dei quali sono rilasciati i master universitari di primo o secondo livello» (Ministero dell'Istruzione dell'Università e delle Ricerca, 2004, art. 3, comma 9).

Essi rilasciano abitualmente dei Diplomi che attestano, di fatto, la frequenza dell'allievo. Come si potrà infatti comprendere dall'Immagine 1 i master fanno parte del sistema formativo nell'ambito degli studi universitari ma non consentono l'acquisizione di un titolo (come la laurea) o di una qualifica (come nel caso delle specializzazioni); non sono inseriti quindi in un processo di sviluppo verticale della formazione universitaria ma rappresentano quel punto d'unione tra mondo accademico e mondo del lavoro che consente una formazione specialistica e frutto delle esigenze dirette del territorio e questo li rende un ibrido difficilmente collocabile.

Infatti essi presentano alcune criticità quali la loro collocazione nel Quadro Nazionale dei Titoli Italiani dell'Istruzione Superiore con riferimento al quadro più ampio tracciato in sede europea. I Master sono dei percorsi di apprendimento organizzati dalle università per rispondere a specifiche esigenze di formazione permanente, il Quadro Nazionale dei Titoli li colloca in tale ambito, separandoli dal percorso accademico sequenziale, di primo, secondo e terzo ciclo, previsto dall'architettura del Processo di Bologna, che rilascia titoli a carattere nazionale. I Master sono gli unici titoli che non figurano nell'elenco dei titoli rilasciati dalle Università di cui ai commi 1 e 2 dell'art. 3 del D.M. n. 270/2004. Gli scarni riferimenti ad essi sono contenuti solo nel comma 9 del suddetto articolo 3. Le incertezze generate da questo scarno sistema di riferimenti normativi sembrava potessero essere superate in sede di prima bozza del decreto di riforma del D.M. n. 509/99, in particolare art. 3, comma 8 del D.M. n. 509/99, dalle quali traspariva l'intenzione di dare una più esplicita valenza formale ai Master, anche per contrapposizione con la formazione che non conduce al conseguimento di un titolo riconosciuto. Per la formazione permanente e ricorrente si prevedeva, infatti, un duplice sbocco: se si fosse tradotta in almeno $60 \mathrm{CFU}$ (Crediti Formativi Universitari), l'Università avrebbe rilasciato il «Diploma» di Master universitario (di I e di II livello); se invece lo studente avesse totalizzato un ammontare di crediti minore, gli sarebbe stato rilasciato solo un "Attestato" dei crediti formativi universitari acquisiti (art.3, co. 8). Sembra che tale precisione, 


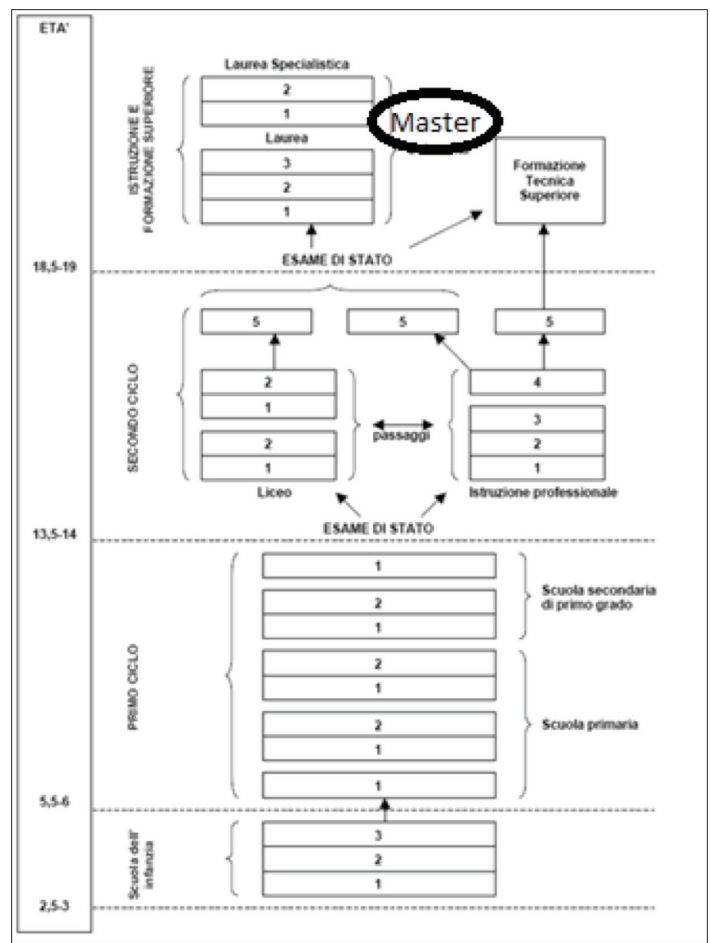

Immagine 1: Struttura Sistema Scolastico Italiano

che peraltro dava rilievo anche all'attestato in sé che lo studente avrebbe potuto inserire nel proprio curriculum, ed in particolare il riconoscimento al Master della natura giuridica formale di diploma, potesse fugare i dubbi sulla natura del Master quale titolo rilasciato dall'Università. Queste modifiche non compaiono nel testo definitivo del D.M. n. 270/2004: di conseguenza la natura del Master Universitario è rimasta quella ambigua già delineata dal D.M. n. 509/99.

In secondo luogo, non va trascurata la forte ambiguità terminologica dei master in ambito europeo, che costituisce un problema molto serio. Vengono chiamati «master» dei percorsi di apprendimento permanente, mentre il resto d'Europa chiama master il secondo ciclo ufficiale del processo di Bologna. Nella collaborazione tra atenei italiani e partner europei si crea quindi un'ambigua commistione tra due percorsi di natura diversa, soprattutto quando si tratta di organizzare dei corsi di studio integrati con titoli congiunti.

In terzo luogo, non si può ignorare che $\mathrm{i}$ master, essendo dei percorsi autonomi, flessibili e svincolati da qualsiasi verifica esterna, non abbiano sempre offerto sufficienti garanzie di qualità. Occorre che, in attesa della valutazione esterna della costituenda agenzia ANVUR, negli atenei stessi ci sia piena consapevolezza che anche i master devono essere sottoposti ad una rigorosa valutazione interna, al fine di garantirne la qualità. 


\section{Il Progetto "Master Universitari e Compe- tenze Acquisite. Validazione e Certificazione": obiettivi, strumenti e risultati}

\section{Il contesto e le domande di ricerca}

Nel Regolamento per la disciplina dei corsi per master universitari dell'Università di Verona che recepisce, come tutti gli atenei italiani, le disposizioni Ministeriali (Ministero dell'Istruzione dell'Università e delle Ricerca, 2004, art. 10, comma 4e) si evidenzia, ad esempio:

I corsi per master sono comprensivi di attività didattica frontale e/o di attività di laboratorio e di altre forme di addestramento, di studio guidato e di didattica interattiva di livello adeguato al grado di perfezionamento che si intende perseguire, integrate da periodi di attività pratica, di stage o dalla redazione di un progetto o elaborato (Università di Verona, 2007, pag. 1).

La peculiarità di questi percorsi è quella di essere «a latere» dei percorsi formativi universitari e quindi quella di muoversi necessariamente a cavallo tra ambiente formale e le richieste del territorio in termini di professionalizzazione (quindi non formale), tra metodologie didattiche classiche e l'experiential learning (Kolb, 1984) favorendo nei partecipanti il raggiungimento dei diversi gradi della conoscenza attraverso il contatto con la realtà professionale stessa e la sua trasformazione attiva (Bion, 1962).

Gli Stage/Project work, sempre presenti in questi percorsi, forniscono quindi agli allievi una possibilità di apprendimento importante e reale, ma: quanto vengono valutati e quindi certificati questi apprendimenti? I processi di valutazione adoperati sono coerenti con la necessità di rilevazione di competenze apprese direttamente dalla pratica lavorativa? Gli standard di certificazione consentono all'allievo di portare sul mercato e testimoniare le competenze così acquisite? Da queste domande siamo partiti in un processo di ricostruzione delle metodologie legate alla didattica, alla valutazione e alla certificazione utilizzate per comprendere i reali modelli formativi sottesi e, con i 13 Master che hanno partecipato al progetto, abbiamo lavorato attivamente alla realizzazione di riprogettazioni degli stessi finalizzate alla comprensione delle competenze richieste dal mercato e derivabili unicamente dalle forme di workplace learning (Billet, 2004) per favorire il cambiamento della struttura di una tipologia di percorso che presenta, a tutt'oggi, forti legami con la didattica accademica finalizzata allo sviluppo delle conoscenze e favorire il passaggio alla didattica per competenze.

\section{Destinatari e la ripartizione dei master coinvolti} Il valore di questo progetto, durato due anni, è legato alla partecipazione di referenti del territorio di diversa natura e del mondo accademico. Infatti, sono stati coinvolti Delegati del Rettore/Prorettori in quanto referenti diretti degli atenei, Presidi di Facoltà che erogano Master Universitari, in quanto respon- 
sabili istituzionali del riconoscimento, delle approvazioni e dell'avvio dei Master, Direttori Scientifici, in quanto responsabili dei contenuti, dei programmi e della valutazione di Master, Comitati Scientifici dei Master, in quanto partecipano attivamente alle fasi progettuali e realizzative dei Master, Coordinatori Didattici, in quanto permettono il raccordo tra i contenuti, lo staff e i partecipanti dei Master, Funzionari amministrativi dedicati ai Master ed alla gestione della formazione post lauream in generale, in quanto coinvolti in tutte le fasi gestionali, Tutor d'aula, in quanto a contatto diretto con i partecipanti, con i docenti e coinvolti nella gestione dei contatti con le aziende ospitanti la fase di stage formativo, Tutor aziendali, in quanto affiancano gli studenti di master nella fase professionalizzante dello stage, Referenti aziendali identificabili come rappresentanti delle figure professionali in uscita dai master ed infine, studenti/ allievi/uditori dei master oggetto di sperimentazione per un totale di 190 soggetti.

I tredici Master, derivanti dall'Università di Verona, dall'Università di Padova e dalla Cà Foscari di Venezia sono stati raggruppati tenendo conto della natura fortemente professionalizzante dei percorsi che sarebbero stati analizzati, al fine di poter lavorare su aree di sbocco occupazionale cercando di favorire il confronto tra i diversi master.

Ragionando in tal senso si è proceduto a catalogare ciascun master per area di riferimento e si sono individuate 5 Aree principali:

- Area Umanistica/Socio Assistenziale (Soc-Ass): Master in Mediazione Familiare e Master in esperto per la disabilità sensoriale.

- Area Organizzativa (Org): è questa l'area più ampia e varia perché racchiude figure che si occupano in senso più generale di organizzazione anche se con ambiti di applicazioni diversi. I master che lo compongono sono: "Cultura del cibo e del vino per la gestione delle risorse enogastronomiche «; «Human Resources Management»; "Diritto del Lavoro»; «Regolazione dello sviluppo locale».

- Area Medica (Med): «Gestione della qualità e della sicurezza del paziente «; «Gestione del rischio clinico e della sicurezza del paziente»; «Management per coordinatori delle professioni sanitarie».

- Area Aziendale (Az): «Business Intelligence e Knowledge Management»; «Internal Auditing».

- Area Informatica (Inf): «Computer game development»; «Tecniche economia e gestione delle comunicazioni e dei media: Mediacom».

Tale organizzazione ci ha consentito di poter lavorare non solo con maggiore senso di collaborazione tra i percorsi aderenti al progetto ma anche di favorire la diffusione delle informazioni. Non si è quindi lavorato più su momenti di incontro singoli ma il lavoro è stato organizzato su «due tornate», la prima d' incontro, socializzazione con i concetti principali da affrontare e prima rilevazione e la seconda di approfondimento ed analisi ragionata sulla base dei primi risultati di riprogettazione. 
La metodologia utilizzata e gli obiettivi del progetto Sulla base dei dati raccolti nella prima fase di analisi della normativa effettuate in fase di progettazione e analizzando i pochi casi studio di rilevazione delle competenze (praticamente nulli risultano essere gli esempi di certificazione) nei master universitari, preso atto delle indicazioni fornite dalla Regione Veneto in termini di Sintassi per l'analisi delle competenze, che si muove nel rispetto dei criteri stabiliti per EQF, European Qualification Framework (Parlamento Europeo 2008) e dell'applicazione degli Indicatori di Dublino si è ritenuto opportuno procedere con un impianto metodologico legato alla RICERCA-AZIONE.

Ricera - Azione è una metodologia di ricerca soprattutto, ma non esclusivamente, qualitativa: questo è vero in particolare nelle prime battute del processo di ricerca quando viene interrogata la domanda, nel corso della cocostruzione di una comune visione del problema che ha originato la domanda conoscitiva, nonché di un comune oggetto sul quale riflettere insieme, sino alle fasi finali di valutazione dell'intero processo nel corso del quale continua a essere richiesta una pratica riflessiva, un continuo esercizio di azione riflessiva cooperativa. Il processo che si mette in atto genera ipotesi provvisorie continuamente rinegoziabili e rinegoziate all'interno del gruppo di lavoro. (Piccardo, Benozzo, 2010, pag. 12) La ricerca-azione è quindi un progetto sociale rispecchiato in un progetto scientifico, un tentativo di mantenere strettamente connessi tra loro il versante di natura sociale/politico/ culturale e quello di natura metodologica.

Il disegno della ricerca non viene quindi predefinito dal ricercatore, ma emerge necessariamente a più livelli e rispetto a molteplici dimensioni: l'oggetto stesso di ricerca ma anche i componenti stessi del gruppo dei partecipanti attivi nella ricerca che rappresentano il tessuto sociale e l'area di sviluppo dell'oggetto di ricerca, le transizioni di sviluppo, l'abilità dei ricercatori.

La Ricerca-Azione viene quindi a delinearsi come un processo di cambiamento ciclico e ricorsivo secondo l'immagina di una spirale ciclica di riflessione critica sull'azione. "L'azione/cambiamento può coincidere anche con la ristrutturazione di pensieri e rappresentazioni degli attori, il riposizionamento rispetto a problemi, lo sviluppo di nuove possibilità di azione, l'assunzione di nuove responsabilità.» (Piccardo, Benozzo, 2010, pag. 207)

Quindi, questo tipo di approccio metodologico implica un atteggiamento prasseologico (legato al contesto ed alla pratica) da parte del ricercatore, comporta una elaborazione di transizioni nella ricerca dell'efficacia per operare delle scelte, richiede l'elaborazione di tattiche, strategie che consentano l'emancipazione degli attori coinvolti finalizzato alla formazione al cambiamento in relazione a problemi sociali attraverso un approccio interdisciplinare.

La rappresentazione della struttura di questa metodologia di ricerca e di lavoro finalizzata alla creazione di nuovi modelli e prassi è sintetizzata nella Immagine 2: Immagine 2: Cicli della Ricerca Azione, da Dalla formazione alla Ricerca azione (http://slideplayer.it/slide/190999/) 


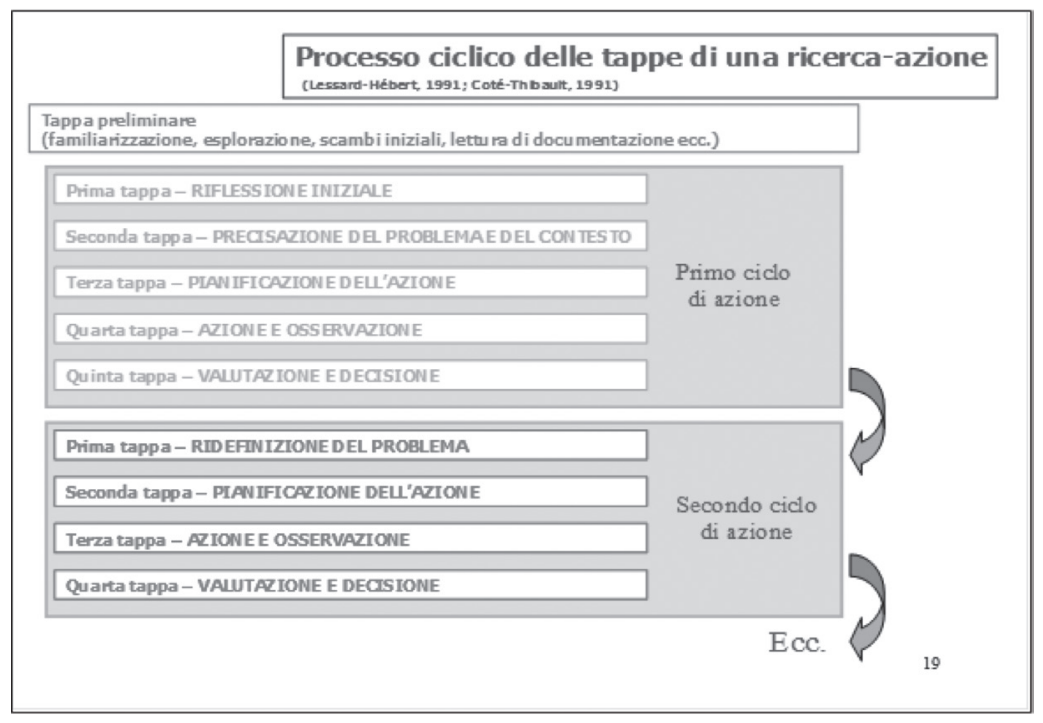

Questo impianto metodologico ha permesso di rispettare appieno lo spirito della Direttiva ed il progetto che si è proposto di descrivere i risultati di apprendimento nell'ambito formale di apprendimento dell' 'istruzione superiore universitaria quale è il Master Universitario di Primo e Secondo Livello e di mettere a punto e sperimentare modelli di riconoscimento, validazione e certificazione delle competenze acquisite con l'obiettivo di:

- realizzare una ricerca scientifica per la creazione di strumenti di identificazione, rilevazione e misurazione delle competenze, in particolare di dispositivi con caratteristiche metodologiche che permettano: il riconoscimento e l'attestazione delle competenze individuali affinché siano messe in relazione con $\mathrm{i}$ titoli e le qualifiche erogati; la verifica del possesso delle competenze in modo corretto; la leggibilità dei contenuti delle competenze misurati nell'ottica della trasparenza dei linguaggi condivisi;

- mettere a punto strumenti di identificazione, rilevazione e misurazione dei risultati di apprendimento e delle competenze acquisite nei master universitari;

- condividere i risultati raggiunti con i partner coinvolti nel progetto, con le Parti Interessate e gli Stakeholders;

- validare gli strumenti realizzati;

- diffondere la cultura del riconoscimento e certificazione delle competenze in ambiti di apprendimento formale; 
- valorizzare e confrontare con quanto emerso in ricerche precedenti sugli studenti e i laureati;

- confrontare l'esistente per evidenziare quanto possa essere trasferito nei modelli di istruzione superiore degli Atenei veneti;

- perfezionare quanto emerso alla luce dei confronti con il sistema di apprendimento locale;

- organizzare tavoli di lavoro e momenti di raccolta dati con i partner del territorio per la condivisione del processo di ricerca;

- comunicare verso l'esterno e l'interno degli Atenei i risultati raggiunti per permettere una condivisione della conoscenza relativa alla certificazione delle competenze.

Non essendoci per i Master una precedente applicazione dei descrittori di Dublino sancita per legge abbiamo ritenuto utile utilizzare un approccio bottom-up, partendo dalla percezione dei direttori di Master secondo una scheda che riportasse le caratteristiche di Conoscenze, Abilità e Competenze secondo quanto definito da EQF e recepito dalla Regione Veneto. Nell'ottica di una rilevazione della capacità di tali percorsi di rilevare e certificare le competenze acquisite dagli allievi più che gli insegnamenti abbiamo chiesto di legare ciascuna delle Conoscenze, Abilità e Competenze identificata con gli strumenti di rilevazione/ valutazione adoperati e di identificare il momento del percorso in cui tale caratteristica si realizza. L'obiettivo sarà quello di andare progressivamente a dettagliare l'attività formativa svolta individuando metodologie adoperate e processi sviluppati incrociando tali fattori con i livelli dell'EQF ed i cicli dei Descrittori di Dublino ${ }^{2}$ riconducibile al master oggetto di studio.

\section{La struttura del progetto}

Il progetto è stato caratterizzato da diverse azioni:

- Seminari Informativi: 10 seminari di informazione relativa alla problematica dell'individuazione, della definizione, dello sviluppo e della valutazione dei risultati di apprendimento attesi in uscita dai Master; 10 incontri informativi con gli stakeholders individuati, per condividere un linguaggio comune.

- Focus group: sono stati realizzati due focus group per ciascun' area tematica. I primi focus group hanno consentito una prima esplorazione delle caratteristiche di questi percorsi formativi, cominciando ad utilizzare una terminologia legata alla competenze attese ed al collegamento tra il percorso formativo e la figura professionale in uscita consentendo, di «raccogliere informazioni utili agli obiettivi di ricerca» (Zammuner, 2003, p.13). Per questa prima fase si è utilizzata una interview guide costruita ad hoc e replicata per ciascun'area al fine di consentirne un confronto. A questo primo ciclo di focus group hanno partecipato: direttori dei master, partner che contribuiscono alla realizzazione dei master, docenti dei master, aziende ospitanti stageur. Se ne mostra lo schema nella Tabella 1. 
Tabella 1: Interview Guide primo ciclo Focus Group

\begin{tabular}{|l|l|}
\hline TOPIC GUIDE & QUESTIONING ROUTE \\
\hline $\begin{array}{l}\text { Apertura, giro di } \\
\text { tavolo }\end{array}$ & $\ldots \ldots \ldots \ldots \ldots \ldots \ldots \ldots \ldots \ldots \ldots . \ldots . \ldots \ldots$ \\
\hline $\begin{array}{l}\text { Riflessioni sul } \\
\text { termine competenza }\end{array}$ & $\begin{array}{l}\text { Negli ultimi tempi si usa molto il termine competenza; cosa vi viene in } \\
\text { mente in relazione a questo termine? }\end{array}$ \\
\hline $\begin{array}{l}\text { Competenze e figura } \\
\text { professionale }\end{array}$ & $\begin{array}{l}\text { Aldilà delle conoscenze, quali sono le competenze fondamentali che } \\
\text { riconoscete nella figura professionale cui fate riferimento? }\end{array}$ \\
\hline $\begin{array}{l}\text { Indicatore di compe- } \\
\text { tenze richieste }\end{array}$ & $\begin{array}{l}\text { In cosa (comportamenti, abilità, capacità) riconoscete ed identificate } \\
\text { queste competenze nella figura professionale cui fate riferimento? }\end{array}$ \\
\hline $\begin{array}{l}\text { Valutazione delle } \\
\text { competenze }\end{array}$ & $\begin{array}{l}\text { Quali possono essere secondo voi i modi di valutare queste competenze } \\
\text { richieste? }\end{array}$ \\
\hline $\begin{array}{l}\text { Strumenti di valuta- } \\
\text { zione }\end{array}$ & $\begin{array}{l}\text { Quali possono essere gli strumenti generalizzabili in grado di creare } \\
\text { parametri raffrontabili? }\end{array}$ \\
\hline $\begin{array}{l}\text { Un diploma/certifi- } \\
\text { cazione facilmente } \\
\text { leggibile }\end{array}$ & $\begin{array}{l}\text { L'obiettivo in uscita da questo progetto di ricerca è creare un documento } \\
\text { facilmente leggibile per capire quali sono le competenze delle persone che } \\
\text { hanno conseguito il diploma di master, cosa ne pensate? }\end{array}$ \\
\hline Suggerimenti & $\begin{array}{l}\text { Sulla base della vostra esperienza quali sono le competenze principali che } \\
\text { dovrebbe contenere? }\end{array}$ \\
\hline Dimenticanze & $\begin{array}{l}\text { C’è qualcosa di cui non abbiamo parlato e che potrebbe essere utile tenere } \\
\text { presente nella'analisi delle competenze in relazione alla figura profes- } \\
\text { sionale? }\end{array}$ \\
\hline
\end{tabular}

Il secondo ciclo di focus group è invece stata realizzata al termine della fase di riprogettazione dei master al fine di condividere gli aspetti individuati come salienti, gli obiettivi, le metodologie didattiche individuate, i comportamenti attesi con rappresentati della figura professionale in uscita e/o rappresentanti del mercato di riferimento nonché gli ex allievi del master (da non più di due anni) che quindi erano in grado di comprendere gli eventuali "gap" tra l'offerta formativa del master e le più recenti richieste del mercato. In tal caso le schede utilizzate sono state collegate direttamente ai singoli master (output rappresentato in Tab. 4) con la richiesta al conduttore di guidare una riflessione tra università e mondo del lavoro sull'efficacia di una riprogettazione così costruita finalizzata ad una figura professionale coerente con il mercato e le necessità di certificazione.

- Workshop: Anche in questo caso l'organizzazione è avvenuta su due cicli, essi sono stati finalizzati alla stesura dettagliata dei learning outcomes di ogni insegnamento preso in analisi. Il primo ciclo d'incontri è stato finalizzato alla schematizzazione delle informazioni recuperate in precedenza attraverso il primo ciclo di focus group, l'analisi delle figure professionali secondo le indicazioni ISFOL 3 e la mappatura dell'offerta formativa dei master coinvolti secondo le informative predisposte per il piano didattico degli atenei (come riportato in Tabella 2). Questa è stata l'occasione per cominciare ad associare 
gli obiettivi formativi dei master ad una logica per Quadro Europeo delle Qualifiche (QEQ) e Descrittori di Dublino.

Tabella 2 Schema di sintesi delle prime informazioni al fine di favorire la riprogettazione dei Master

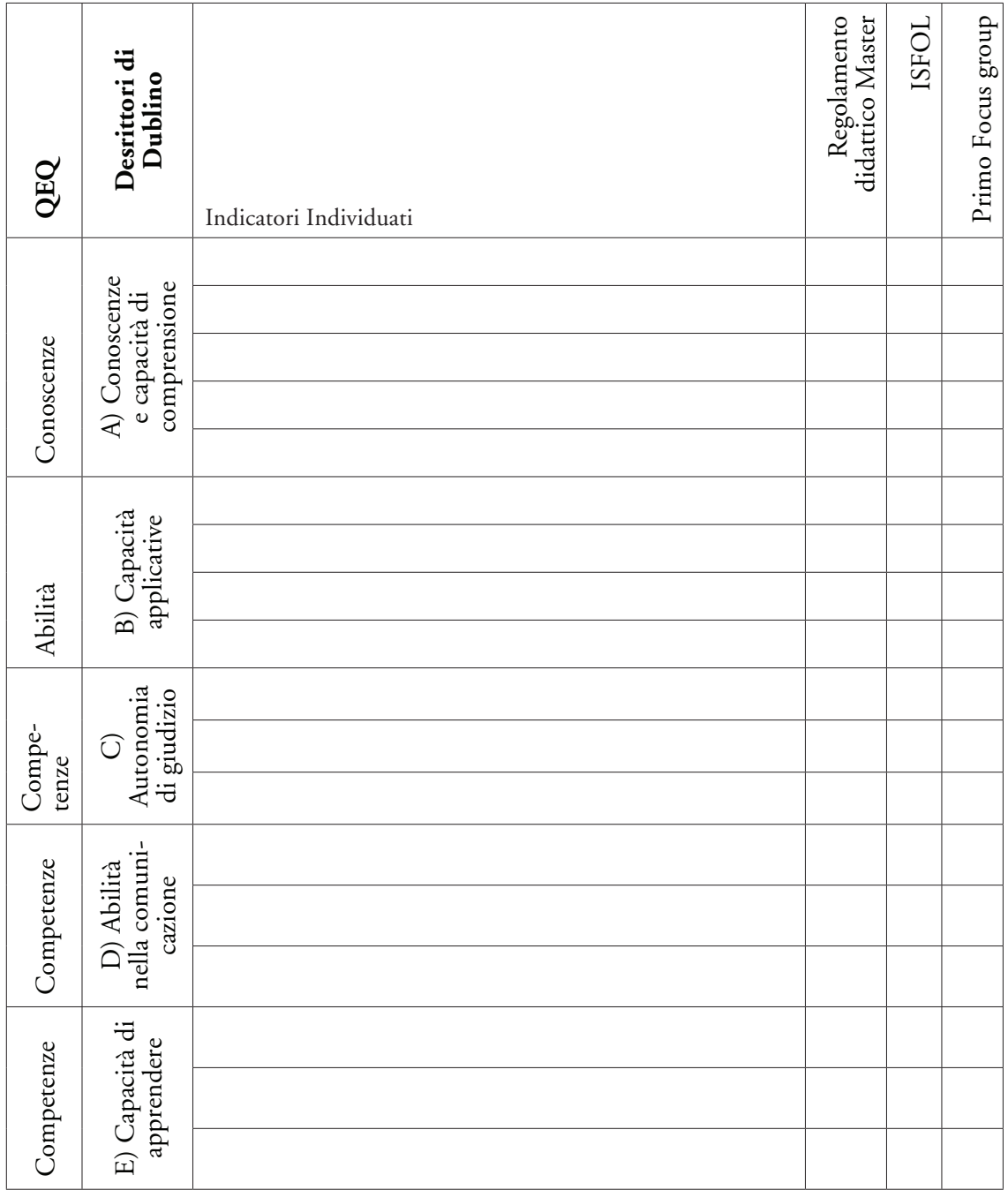

A seguito di questo primo ciclo d'incontri si è provveduto all'individuazione di un consulente esperto che ha seguito, attraverso incontri centrati su singolo Master, alla stesura di una prima definizione dei comportamenti attesi coerenti con le informazioni emerse, declinati sulla base dei Descrittori di Dublino e 
coerentemente con la possibilità di individuare metodologie didattiche applicate e relativi strumenti di valutazione.

Attraverso la consulenza del coach si è quindi pervenuti alla descrizione di ciascun master rispecchiando la seguente Tabella 3:

Tabella 3 Schema riprogettazione Master

\begin{tabular}{|c|c|c|c|c|c|c|}
\hline \multirow[b]{2}{*}{ Modulo } & \multirow[b]{2}{*}{$\begin{array}{c}\text { Area di } \\
\text { Compe- } \\
\text { tenza }\end{array}$} & \multirow{2}{*}{$\begin{array}{c}\text { Conoscenze } \\
\text { Conoscenze } \\
\text { e capacità } \\
\text { di compren- } \\
\text { sione }\end{array}$} & \multicolumn{4}{|l|}{ Abilità } \\
\hline & & & $\begin{array}{l}\text { Capacità } \\
\text { applicative }\end{array}$ & $\begin{array}{l}\text { Autonomia } \\
\text { di giudizio }\end{array}$ & $\begin{array}{c}\text { Abilità } \\
\text { nella } \\
\text { comunica- } \\
\text { zione }\end{array}$ & $\begin{array}{l}\text { Capacità di } \\
\text { apprendere }\end{array}$ \\
\hline & & $\begin{array}{l}\text { Comporte- } \\
\text { menti attesi }\end{array}$ & $\begin{array}{l}\text { Compor- } \\
\text { tementi } \\
\text { attesi }\end{array}$ & $\begin{array}{l}\text { Compor- } \\
\text { tementi } \\
\text { attesi }\end{array}$ & $\begin{array}{l}\text { Compor- } \\
\text { tementi } \\
\text { attesi }\end{array}$ & $\begin{array}{l}\text { Compor- } \\
\text { tementi } \\
\text { attesi }\end{array}$ \\
\hline
\end{tabular}

Alla seconda fase di focus group si è quindi fato seguito con un nuovo ciclo di workshop in cui si è provveduto ad una definitiva stesura e confronto dei learning outcomes attesi nei master, all'identificazione delle aree di applicazione della sperimentazione grazie anche all'individuazione delle metodologie didattiche ed ai relativi momenti di valutazione individuati.

Ciascun master è stato quindi descritto secondo la seguente griglia costruita ad hoc di cui riportiamo un esempio esemplificativo per le Conoscenze:

Lungo questa riprogettazione i Seminari hanno mantenuto l'obiettivo di essere momento di approfondimento/in-formazione ma non più specifico per singolo master bensì generalizzato alle richieste condivise dai percorsi. Si sono ad esempio realizzati due incontri (al fine di favorire la massima partecipazione) legati ai temi della certificazione delle competenze ed al senso del cambiamento apportato nel panorama dell'alta formazione dal «Processo di Bologna», un incontro seminariale è stato dedicato alla figura del Tutor quale facilitatore didattico e supporto alla realizzazione di un processo di valutazione e di orientamento adeguato. Vista inoltre l'importanza delle metodologie didattiche attive più momenti seminariali sono stati dedicati all'esplicitazione del legame tra metodologie didattiche specifiche ed acquisizione di competenze. Attraverso questi incontri si è cercato di illustrare l'esistenza di metodologie didattiche attive alternative/innovative come l'action learning. 
Tabella 4 (esempio di outpout di riprogettazione del Master "Regolazione dello sviluppo locale» Università di Pavova A.A. 2011 - 2012)

\begin{tabular}{|c|c|c|c|c|c|c|}
\hline $\begin{array}{l}\stackrel{\partial}{\partial} \\
\text { d }\end{array}$ & 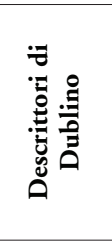 & $\begin{array}{l}\text { Indicatori } \\
\text { Indivi- } \\
\text { duati }\end{array}$ & $\begin{array}{l}\text { Compor- } \\
\text { tamenti } \\
\text { Attesi } \\
\text { negli } \\
\text { allievi: } \\
\text { L'allievo } \\
\text {......... }\end{array}$ & $\begin{array}{l}\text { Definizione sulla } \\
\text { base del Ciclo } \\
\text { del Descrittore } \\
\text { di Dublino }\end{array}$ & $\begin{array}{l}\text { Metodo- } \\
\text { logia } \\
\text { didattica } \\
\text { utile }\end{array}$ & $\begin{array}{c}\text { Strumento } \\
\text { di valuta- } \\
\text { zione } \\
\text { idoneo }\end{array}$ \\
\hline 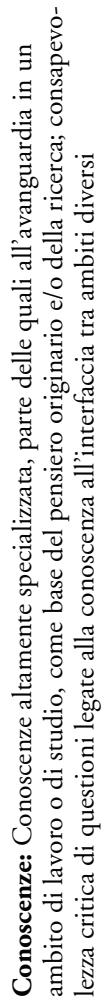 & 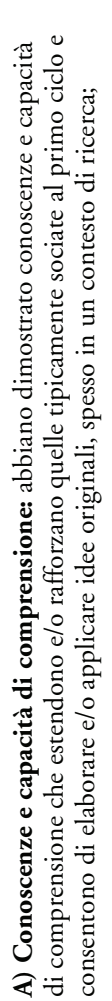 & $\begin{array}{l}\text { Principi e } \\
\text { teorie di } \\
\text { network } \\
\text { gover- } \\
\text { nance }\end{array}$ & $\begin{array}{l}\text { Conoscere } \\
\text { i diversi } \\
\text { tipi di reti } \\
\text { / network }\end{array}$ & $\begin{array}{l}\text { Conoscere la } \\
\text { teoria delle } \\
\text { reti: Analisi dei } \\
\text { contesti locali e } \\
\text { regionali; Analisi } \\
\text { del modello di } \\
\text { regolazione e } \\
\text { sviluppo locale } \\
\text { in prospettiva } \\
\text { europea e globale; } \\
\text { Distinguere: } \\
\text { attori economici, } \\
\text { politici e sociali } \\
\text { che parte- } \\
\text { cipano alle reti } \\
\text { (governance } \\
\text { multiattore), } \\
\text { la cultura ed il } \\
\text { sistema di valori } \\
\text { degli attori, il } \\
\text { contesto istitu- } \\
\text { zionale di riferi- } \\
\text { mento, in una } \\
\text { prospettiva storica } \\
\text { di lungo periodo; } \\
\text { Analisi organiz- } \\
\text { zativa in dimen- } \\
\text { sione europea } \\
\text { (governance } \\
\text { multilivello). }\end{array}$ & $\begin{array}{l}\text { Metodo- } \\
\text { logia } \\
\text { Didattica: } \\
\text { Lezione } \\
\text { frontale, } \\
\text { presen- } \\
\text { tazione } \\
\text { analisi e } \\
\text { discussione } \\
\text { di casi } \\
\text { studio, } \\
\text { ricostru- } \\
\text { zione delle } \\
\text { categorie } \\
\text { utilizzate } \\
\text { in chiave } \\
\text { comparata. }\end{array}$ & $\begin{array}{l}\text { Risposta a } \\
\text { domanda } \\
\text { aperta. }\end{array}$ \\
\hline
\end{tabular}

\section{I risultati dell'approccio per competenze in termini di metodologie didattiche}

I risultati principali di questo progetto, in termini di relazione tra gli elementi di apprendimento formale e non formale risiede, a nostro parere, nell'approccio alla progettazione utilizzato e fin qui illustrato. Lavorando con percorsi così diversi infatti ci si è resi conto che, proprio per il forte collegamento con la parte di stage/project Work è pressoché impossibile definire per tutti un'unica strutturazione per metodologie didattiche e modelli di valutazione ma è possibile utilizzare un modello descrittivo che coniughi i modelli «non formali», derivanti 
dalle attese del mercato del lavoro con le istanze «formali» che necessitano di una strutturazione rigida.

A seguito di questo progetto infatti $\mathrm{i}$ tre atenei aderenti hanno utilizzato un modello unico di descrizione dei master sulla base di una descrizione degli obiettivi formativi declinati secondo i Descrittori di Dublino, ed una richiesta, in fase di progettazione, di rilevazione dei fabbisogni emergenti dal mercato. Due atenei su tre hanno ritenuto utile suggerire, in fase di progettazione del master la compilazione della sceda riportata in Tabella 4.

Attraverso la strutturazione di una griglia costruita ad hoc abbiamo potuto rilevare la metodologia didattica utilizzata per il raggiungimento di ciascuno dei comportamenti attesi nell'allievo. Si è trattato di un percorso particolarmente lungo poiché le metodologie didattiche utilizzate sono appannaggio dei singoli docenti e poiché in media un Master ha un corpo docenti di 15/20 docenti abbiamo avuto necessità di contattare buona parte di essi per un totale di 238 docenti.

A seguito di questo lavoro sono stati sintetizzati gli metodologie utilizzate che vengono riportate con la percentuale di rappresentatività delle stesse sul totale dei master:

\begin{tabular}{|c|c|c|}
\hline Metodologia didattica rilevata & $\begin{array}{l}\text { Prima } \\
\text { rilevazione } \\
25 / 05 / 2010\end{array}$ & $\begin{array}{l}\text { Rilevazione } \\
\text { post ripro- } \\
\text { gettazione } \\
30 / 05 / 2011\end{array}$ \\
\hline \multicolumn{3}{|l|}{ Attività pratica su pazienti/Pratica clinica } \\
\hline $\begin{array}{l}\text { Brain storming (tempesta di idee nel cervello : produzione autonoma } \\
\text { di ipotesi e concetti - attraverso la libera associazione di idee - preli- } \\
\text { minare all'attività del docente) }\end{array}$ & $3(1 \%)$ & $\mathbf{0}(\mathbf{0 \%})$ \\
\hline Cooperative learning (apprendimento collaborativo.) & $1(0 \%)$ & $0(0 \%)$ \\
\hline Dialogico - eromatico (interattività) & $6(3 \%)$ & $6(3 \%)$ \\
\hline E learning (modalità blanded) & $4(2 \%)$ & $4(2 \%)$ \\
\hline Laboratori esperienziali & $2(7 \%)$ & $2(8 \%)$ \\
\hline Visite guidate in realtà del territorio & $1(0 \%)$ & $1(0 \%)$ \\
\hline Peer tutoring (docente facilitatore dell'apprendimento, ecc.) & $2(0 \%)$ & $\mathbf{0}(0 \%)$ \\
\hline Project work & $2(5 \%)$ & $2(4 \%)$ \\
\hline $\begin{array}{l}\text { Role playing (esposizione di relazioni per sequenze di mappe concet- } \\
\text { tuali, drammatizzazione di contenuti e concetti, dibattiti, lavori di } \\
\text { gruppo ecc.) }\end{array}$ & $4(2 \%)$ & $7(3 \%)$ \\
\hline Simulazioni & $1(2 \%)$ & $4(5 \%)$ \\
\hline Stage & $10(28 \%)$ & $10(28 \%)$ \\
\hline Studio di casi & $7(13 \%)$ & $9(11 \%)$ \\
\hline Team-teaching (compresenza di docenti.) & $1(0 \%)$ & $1(0 \%)$ \\
\hline $\begin{array}{l}\text { Unidirezionale (lezione frontale, introduttiva o di raccordo; parteci- } \\
\text { pazione a convegni) }\end{array}$ & $12(37 \%)$ & $12(36 \%)$ \\
\hline
\end{tabular}


In questo caso più che la frequenza sulla presenza delle metodologie didattiche può essere utile concentrarsi sulla percentuale media di utilizzo delle stesse all'interno dei percorsi (dato riportato in parentesi).

Le metodologie attive risultano coprire circa il $60 \%$ delle tipologie di didattica utilizzate ed è possibile rilevare come, a seguito di una riprogettazione per competenze aumenti sensibilmente l'utilizzo di simulazioni e role playng, cioè di quelle metodologie che si legano fortemente allo sviluppo di competenze/ abilità professionali.

\section{Considerazioni Conclusive}

Quanto fin qui illustrato, pur nella consapevolezza di un progetto ristretto ad un territorio circoscritto, porta la consapevolezza che distinguere nettamente tra apprendimenti formali e non formali potrebbe non solo non rispecchiare la pratica formativa contemporanea che necessita invece di percorsi di formazione più flessibili per facilitare le transizioni tra le fasi lavorative e quelle formative in un approccio integrato di contesti formativi allargati, concetto di confini liquidi ed indefiniti (Bauman, 2006) o di società mobile (Beck, 2004) che richiede all'uomo contemporaneo di essere costantemente in formazione continua (sempre e comunque) ma che richiede anche a chi di educazione e formazione si occupa di unire momenti formativi diversi alla costruzione di qualcosa di più complesso dei classici saperi (Alessandrini 2012). I risultati del progetto hanno consentito una riflessione da parte degli atenei coinvolti nella strutturazione di un modello di costruzione e certificazione idoneo e coerente con le aspettative di applicazione del «Diploma supplement»( DM 509 del 1999, articolo 11, comma 8). Ulteriore ricaduta del progetto è stata la formalizzazione in un modello condiviso, a livello regionale, di un modello per la redazione di un dossier delle Evidenze da far confluire all'interno del Libretto Formativo del Cittadino per validazione delle competenze acquisiste lungo tutto l'arco della vita (DGR 2895 del 28/12/2012) in cui sono stati assorbite alcuni degli strumenti utilizzati durante la fase di sperimentazione e comuni ad altri progetti condotti con lo stesso finanziamento.

\section{Note}

1 La dicitura «FSE 2007-2013» identifica la tipologia di fondi strutturali a cui si fa riferimento ed il periodo di finanziamento del fondo (non del progetto). La specifica risulta necessaria sia in termini di obiettivi di finanziamento sia in termini d'identificazione dell'Agenda Europea in cui sono inseriti.

2 Istituiti nel 2004 i Descrittori di Dublino sono definibili come: descrittori dei risultati di apprendimento - learning outcomes» dei cicli per la formazione superiore. I «descrittori di Dublino» sono costruiti sugli elementi seguenti: Conoscenze e capacità di comprensione (knowledge and understanding); Utilizzazione delle conoscenze e capacità di comprensione (applying knowledge and understanding); Capacità di trarre conclusioni (making 
judgements); Abilità comunicative (communication skills); Capacità di apprendere (learning skills). (www.processodibologna.it/documentieuropei)

3 ISFOL: l'Istituto per lo sviluppo della formazione professionale dei lavoratori, istituito nel giugno del 1973. dal 1999 è un ente pubblico di ricerca che si occupa, tra l'altro, della formazione professionale grazie al fatto che opera in stretta collaborazione con l'Unione Europea e tanti organismi internazionali e, in Italia, con le Regioni, le parti sociali, la Presidenza del Consiglio dei ministri, il Ministero dell'istruzione, dell'università e della ricerca ed il Ministero del lavoro, della salute e delle politiche sociali

\section{Bibliografia}

Alessandrini, G. (2012). Comunità di pratica e Società della conoscenza. Roma: Carocci - Le Bussole.

Bauman, Z. (2006). Vita liquida. Roma-Bari: Laterza.

Beck, U. (2004). Il lavoro nell'epoca della fine del lavoro. Tramonto delle sicurezze e nuovo impegno civile. Torino: Einaudi.

Billet, S. (2004). Learning through work: workplace participatory practices. London, England: Routledge.

Bion, W. R. (1962), Apprendere dall'esperienza. Roma, Armando Editore.

Cedefop. (2010). Skills supply and demand in Europe Medium-term forecast up to 2020. Luxemburg: Pubblication Office of European Union

Crocker, D.A, Robeyns, I. (2010). Capability and Agency. In C. W. Morris (Ed.), Amartya Sen. Cambridge: Cambridge University Press.

Commissione delle Comunità Europee. (2000). «Relazione sul memorandum della Commissione sull'istruzione e la formazione lungo tutto l'arco della vita». 485 final. Bruxelles, 5.9.2012. [on line: http://ec.europa.eu/education/lifelong-learning-policy/doc/informal/ proposal2012_it.pdf; consultato il 08 Gennaio 2014]

Commissione Europea. (2012). Raccomandazione del consiglio sulla "convalida dell'apprendimento non formale e informale». 485 final. Bruxelles, 5.9.2012. [on line: http:// eur-lex.europa.eu/LexUriServ/LexUriServ.do?uri=OJ:C:2012:398:0001:0005:IT:PDF; consultato il 09 Gennaio 2014]

Formicuzzi, M., Cubico, S., Ardolino, P., Favretto, G. (2012). Professional Higher Education: From Skills To Diploma Supplement. In: 4th International Conference on Education, Economy and Society. Paris.

Formicuzzi, M., Gadioli, De Oliveira, J., Cubico, S., Ardolino, P., Favretto, G. (2013). Progetto"Master Universitari e competenze acquisite. Validazione e certificazione». Sperimentazione di un processo di valutazione e certificazione di apprendimenti formali in ambito universitario . In: AAVV. Abstract Book. p. 178-179, Lugano, 21- 23 Agosto 2013

Gola, G (2009). L'Apprendimento informale nella professione. Roma, ARACNEeditrice.

ISFOL. (2012a). Primo Rapporto italiano di referenziazione delle qualificazioni al quadro europeo EQF. [on line: http://bw5.cilea.it/bw5ne2/opac.aspx?WEB=ISFL\&IDS=18746; consultato il 15 Gennaio 2014]

Kolb, D. (1984). Experiential learning. Englewood Cliffs: Prentice-Hall.

Midoro, V. (2002). Dalle comunità di pratica alle comunità di apprendimento virtuali, TD -Tecnologie Didattiche, n.25, 1, pp. 3-10, Ortona (CH), Menabò Edizioni [on line:

www.itd.cnr.it/tdmagazine/PDF25/Com_Pratica.pdf; consultato il 10 Gennaio 2014]

Ministry of Science, Technology and Innovation. (2005). A Framework for the European Higher Education Area - QF for the EHEA. [on line: http://www.bologna-bergen2005.no/ Docs/00-Main_doc/050218_QF_EHEA.pdf; consultato il 10 Gennaio 2014]

Ministero dell'Università e della Ricerca Scientifica e Tecnologica (1999). Decreto ministeriale 3 novembre 1999, n.509. [on line: http://www.miur.it/0006Menu_C/0012Docume/ 0098Normat/2088Regola.htm; consultato il 09 Settembre 2014] 
Ministero dell'Istruzione dell'Università e della Ricerca (2004). Decreto Ministeriale 22 ottobre 2004, n. 270. [on line: http://www.miur.it/0006Menu_C/0012Docume/0098Normat/ 4640Modifi_cf2.htm; consultato il 09 Gennaio 2014]

Parlamento Europeo. (2008) Raccomandazione del parlamento europeo e del consiglio del 23 aprile 2008 sulla costituzione del quadro europeo delle qualifiche per l'apprendimento permanente. [on linehttp://ec.europa.eu/eqf/documentation_en.htm; consultato il 10 Gennaio 2014]

Piccardo, C., Benozzo, A. (2010). Verso una definizione complessa di ricerca-azione. In Kaneklin, C., Scaratti, G., Piccardo, C. (a cura di), La Ricerca-Azione nelle organizzazioni (pp. 1-28). Milano: Cortina Editore.

Regione Veneto. (2012). Linee guida per la validazione di competenze acquisite in contesti non formali ed informali. DGR 2895 del 28/12/2012». [on line:

http://www.regione.veneto.it/c/document_library/get_file?uuid=25b337e3-47b5-49c4ba34-877709d85c21\&groupId=60642; consultato il 10 Gennaio 2014]

Sen, A.K. (1993). Capability and Well-Being. in M.C. Nussbaum and A.K. Sen (eds), The Quality of Life, Oxford: Clarendon Press.

Sen, A.K. (1999). Development as Freedom. Oxoford: Oxoford University Press.

Senato della Repubblica Italiana. (2010). Decreto Legge 137/200. [on line:

http://www.senato.it/japp/bgt/showdoc/frame.jsp?tipodoc=Ddlpres\&leg=16\&id=513824; consultato il 10 Gennaio 2014]

Trentin, G. (2000). Dalla formazione a distanza alle comunità di pratica attraverso

l'apprendimento in rete, TD - Tecnologie Didattiche, n.20, 2, pp.21.29, Ortona (CH), Menabò Edizioni [on line: www.itd.cnr.it/TDMagazine/PDF20/Trentin.pdf; consultato il 08 Gennaio 2014]

Università di Verona (2007). Regolamento per la disciplina dei corsi per master universitari. [on line: http://www.univr.it/documenti/Documento/allegati/allegati752587.pdf; consultato il 15 Gennaio 2014]

Viganò, R. (2008). Dalla formazione alla ricerca-azione Una sperimentazione efficace nella scuola. http://slideplayer.it/slide/190999/ Consultato il 12 gennaio 2014

Zammuner, V.L. (2003). I focus Group. Aspetti della psicologia. Bologna: Il Mulino

Parole chiave: Apprendimenti formali, apprendimenti non formali, certificazione competenze, master universitari.

\section{Hochschulausbildung zwischen formalem und nicht-formalem Lernen: Das Beispiel Master an italienischen Universitäten}

\section{Zusammenfassung}

Die Realisierung des vorliegenden Forschungsprojekts "Master Universitari e competenze acquisite: Validazione e certificazione» wird durch die Region Veneto finanziert. Dieses Projekt hat uns erlaubt, eine Reihe von Beobachtungen und Reflexionen zu Lehrmethoden und Instrumenten der Evaluierung und Zertifizierung bezüglich des Lernens im formalen als auch nicht-formalen Bereich zu reflektieren.

Schlagworte: Formales und nicht-formales Lernen; Zertifizierung, Evaluierung von Kompetenzen, Zertifizierung von Kompetenzen, Hochschulbildung. 


\section{La formation professionnelle supérieure entre apprentissage formel et non formel: l'exemple du Master dans les universités italiennes}

\section{Résumé}

Le présent travail s'inspire de la réalisation du projet de recherche et d'intervention "Master Universitari e competenze acquisite: Validazione e certificazione», financé par la Région de la Vénétie. Ce projet nous a permis de formuler un certain nombre d'observations et de réflexions sur les méthodes d'enseignement et sur les outils d'évaluation et de certification liés aux apprentissages réalisés à la fois en contextes formel et informel.

Mots-clés: Apprentissage formel, apprentissage non formel, évaluation des compétences, certification des compétences, formation professionnelle universitaire.

\section{High vocational training between formal and non-formal learning: The case of Master programs in Italian Universities}

\section{Summary}

The present work results from the realization of the research and intervention project "Master Universitari e competenze acquisite: Validazione e certificazione» funded by the Veneto Region. This project has allowed us to achieve a number of observations and reflections on teaching methodologies, assessment and certification tools related to learning achieved in the field of both formal and non-formal learning.

Keywords: Formal learning, non-formal learning, competency assessment, competency certification, university vocational training. 
